\title{
Association Study Between SLC15A4 Polymorphisms and Haplotypes and Systemic Lupus Erythematosus in a Han Chinese Population
}

\author{
Mingwang Zhang, ${ }^{1}$ Fangru Chen, ${ }^{2}$ Dongmei Zhang, ${ }^{1}$ Zhifang Zhai, and Fei Hao ${ }^{1}$
}

Objective: The gene SLC15A4 (solute carrier family 15 [oligopeptide transporter], member 4) has been reported as contributing to the pathogenesis of systemic lupus erythematosus (SLE). We performed a case-control replication study to investigate further the association between single-nucleotide polymorphisms (SNPs) in the SLC15A4 gene and systemic SLE in a Han Chinese population. Methods: In Han Chinese SLE patients and healthy individuals ( $n=355,375$, respectively), 18 SNPs in the SLC15A4 gene were genotyped using matrix-assisted laser desorption/ ionization time-of-flight mass spectrometry and TaqMan SNP genotyping assays. Analyses of allele frequencies and genotypes using codominant, dominant, and recessive models were conducted, as well as a linkage disequilibrium analysis. $P$ values $<0.05$ were considered significant. Results: Allele frequencies of five of the analyzed SNPs were significantly associated with SLE. Under a codominant model the genotype frequencies of rs3765108 AG and rs7308691 AT were significantly higher in the SLE group than the control group ( $p=0.019,0.049$, respectively). Under a dominant model the rs1385374 (TT+CT) SNP carried a higher risk of SLE than (CC) $(p=0.042)$. One SLC15A4 haplotype (TA), which consists of 2 SNPs (rs959989 and rs983492), was associated with SLE ( $p=0.024)$. Conclusion: Our study determined that five SNPs (rs959989, rs1385374, rs983492, rs12298615, and rs10847697) are associated with SLE. Thus, SLC15A4 may be important in the pathogenesis of SLE in Han Chinese patients.

\section{Introduction}

$\mathbf{S}$ YSTEMIC LUPUS ERYTHEMATOSUS (SLE) is a complex autoimmune disease with multiple organ injury. SLE is characterized by aberrant $\mathrm{T}$ - and B-cell activation, leading to upregulation of autoantibody production and loss of immunological tolerance to self-nuclear antigens. The etiology of SLE is complex and involves the interaction of genetic, epigenetic, environmental, hormonal, and immunoregulatory factors (Tsokos, 2011). In recent years, the genes causing SLE have been identified, with more than 40 susceptible loci, by using targeted, genome-wide linkage analysis and, especially, genome-wide association studies (GWASs).

SLC15A4 (solute carrier family 15 , member 4), is a protoncoupled histidine and oligopeptide cotransporter that contains 12 membrane-spanning domains. SLC15A4 is predominantly transcribed in the brain and immune cells, and especially in plasmacytoid dendritic cells (Yamashita et al., 1997; Sasawatari et al., 2011; Nakamura et al., 2014). Through research with SLC15A4-mutant or SLC15A4deficient mice, lack of SLC15A4 has been linked to impaired nucleotide oligomerization domain-1 (NOD1)-, toll-like receptor 7 (TLR7)-, and TLR9-dependent cytokines such as type 1 interferon (IFN), all of which are indispensable to the pathogenesis of SLE (Sasawatari et al., 2011; Baccala et al., 2013; Kobayashi et al., 2014).

Recent GWASs reported that SLC15A4 is a lupusassociated locus in both the Chinese and Korean populations (Han et al., 2009; Lee et al., 2014). A genotype-phenotype analysis indicated that the single-nucleotide polymorphism (SNP) rs10847697 of the gene has a stronger genetic effect on SLE patients with discoid rash in Han Chinese people (He et al., 2010). Another group showed that the SNPs rs 10847697 and rs 1385374 of SLC15A4 were significantly associated with renal involvement in SLE in Caucasian populations (Wang et al., 2013). All of these observations indicate that mutants or polymorphisms of SLC15A4 have an indispensable role in the pathogenesis of SLE.

To our knowledge, previous studies have focused only on the two SNPs identified from the GWASs. However, the GWASs genotyped hundreds of thousands of tag-SNPs using the Illumina platform, which demands a highly significant

\footnotetext{
${ }^{1}$ Department of Dermatology, Southwest Hospital, Third Military Medical University, Chongqing, China.

${ }^{2}$ Department of Dermatology, Affiliated Hospital of Guilin Medical College, Guilin, China.

(c) Mingwang Zhang et al., 2016; Published by Mary Ann Liebert, Inc. This Open Access article is distributed under the terms of the Creative Commons License (http://creativecommons.org/licenses/by/4.0), which permits unrestricted use, distribution, and reproduction in any medium, provided the original work is properly credited.
} 
$p$-value, and therefore, some SNPs associated with SLE may have been missed.

In the present study, we evaluated associations between SLC15A4 polymorphisms and SLE in southwestern Han Chinese people through comparisons between 355 SLE patients and 375 healthy individuals. In particular, we focused on the association of alleles and genotypes of 18 SNPs with SLE, and a linkage disequilibrium analysis of these SNPs.

\section{Materials and Methods}

The local regional ethics committee approved the study. All participants provided written informed consent.

\section{Patients and controls}

A case-control study was performed with 355 SLE patients (10 men and 345 women, mean age $32.3 \pm 12.5$ years) and 375 healthy individuals (control group; 18 men and 357 women, mean age $34.0 \pm 7.6$ years). All subjects were from the
Chongqing, Sichuan, Yunnan, or Guizhou areas of southwestern China and are of Han Chinese ethnicity. Patients were recruited from the Department of Dermatology, Southwestern Hospital of the Third Military Medical University (Chongqing, China) from January 2008 to March 2013. All patients with SLE had fulfilled the revised diagnostic criteria of the American College of Rheumatology (1997) and excluded other systematic or autoimmune diseases (Hochberg, 1997). Blood samples from the control group were collected at the Medical Examination Center of the same hospital during regular health examinations. All of the healthy individuals were free of history or symptoms of SLE or other autoimmune diseases.

\section{DNA samples and genotyping assays}

Venous peripheral blood $(2 \mathrm{~mL})$ was collected from each participant with ethylenediaminetetraacetic acid anticoagulant and stored at $-80^{\circ} \mathrm{C}$ until used. Genomic DNA was isolated using a genomic DNA extraction kit (Sangon Biotech) in accordance with the manufacturer's instructions.

Table 1. Distribution of Allele Frequencies in Single-Nucleotide Polymorphisms of SLC15A4 in Systemic Lupus ERythematosus Patients and Controls

\begin{tabular}{|c|c|c|c|c|c|c|c|}
\hline \multirow[b]{2}{*}{ SNP } & \multirow[b]{2}{*}{ Allele } & \multicolumn{2}{|c|}{$\operatorname{SLE}(\mathrm{n}=355)$} & \multicolumn{2}{|c|}{ Control $(\mathrm{n}=375)$} & \multirow[b]{2}{*}{$O R(95 \% C I)$} & \multirow[b]{2}{*}{$\mathrm{P}$} \\
\hline & & Count & $\%$ & Count & $\%$ & & \\
\hline \multirow[t]{2}{*}{ rs7965732 } & A & 642 & 90.42 & 671 & 89.47 & Reference & \\
\hline & $\mathrm{T}$ & 68 & 9.57 & 79 & 10.53 & $0.9(0.639,1.266)$ & 0.544 \\
\hline \multirow{2}{*}{ rs3765108 } & $\mathrm{A}$ & 479 & 67.46 & 512 & 68.27 & Reference & \\
\hline & $\mathrm{G}$ & 231 & 32.54 & 238 & 31.73 & $1.037(0.833,1.292)$ & 0.743 \\
\hline \multirow[t]{2}{*}{ rs7308691 } & $\mathrm{A}$ & 233 & 32.91 & 237 & 31.77 & Reference & \\
\hline & $\mathrm{T}$ & 475 & 67.09 & 509 & 68.23 & $0.949(0.762,1.183)$ & 0.642 \\
\hline \multirow[t]{2}{*}{ rs7302634 } & $\mathrm{C}$ & 307 & 43.48 & 322 & 42.93 & Reference & \\
\hline & $\mathrm{T}$ & 399 & 56.52 & 428 & 57.07 & $0.978(0.795,1.203)$ & 0.832 \\
\hline \multirow[t]{2}{*}{ rs9738216 } & $\mathrm{C}$ & 307 & 43.36 & 321 & 42.91 & Reference & \\
\hline & $\mathrm{T}$ & 401 & 56.64 & 427 & 57.09 & $0.982(0.798,1.208)$ & 0.863 \\
\hline \multirow[t]{2}{*}{ rs2291350 } & $\mathrm{C}$ & 309 & 43.64 & 322 & 42.93 & Reference & \\
\hline & $\mathrm{T}$ & 399 & 56.36 & 428 & 57.07 & $0.971(0.790,1.195)$ & 0.784 \\
\hline \multirow[t]{2}{*}{ rs11059915 } & $\mathrm{G}$ & 546 & 76.9 & 562 & 74.93 & Reference & \\
\hline & $\mathrm{T}$ & 164 & 23.1 & 188 & 25.07 & $0.898(0.706,1.142)$ & 0.38 \\
\hline \multirow{2}{*}{ rs12298615 } & A & 169 & 31.35 & 147 & 19.6 & Reference & \\
\hline & $\mathrm{G}$ & 539 & 68.65 & 603 & 80.4 & $0.778(0.606,0.998)$ & $0.048^{\mathrm{a}}$ \\
\hline \multirow[t]{2}{*}{ rs4760592 } & $\mathrm{C}$ & 401 & 56.48 & 429 & 57.35 & Reference & \\
\hline & $\mathrm{G}$ & 309 & 43.52 & 319 & 42.65 & $1.036(0.842,1.275)$ & 0.736 \\
\hline \multirow[t]{2}{*}{ rs959989 } & A & 537 & 75.63 & 601 & 80.35 & Reference & \\
\hline & $\mathrm{T}$ & 173 & 24.37 & 147 & 19.65 & $1.317(1.027,1.689)$ & $0.03^{\mathrm{a}}$ \\
\hline \multirow[t]{2}{*}{ rs959987 } & $\mathrm{C}$ & 578 & 81.64 & 580 & 77.54 & Reference & \\
\hline & $\mathrm{T}$ & 130 & 18.36 & 168 & 22.46 & $0.776(0.601,1.003)$ & 0.053 \\
\hline \multirow[t]{2}{*}{ rs7311875 } & A & 537 & 76.06 & 559 & 74.53 & Reference & \\
\hline & $\mathrm{G}$ & 169 & 23.94 & 191 & 25.47 & $0.921(0.726,1.169)$ & 0.499 \\
\hline \multirow[t]{2}{*}{ rs11059925 } & A & 558 & 80.64 & 593 & 79.07 & Reference & \\
\hline & $\mathrm{C}$ & 134 & 19.36 & 157 & 20.93 & $0.907(0.701,1.174)$ & 0.458 \\
\hline \multirow{2}{*}{ rs10847697 } & A & 173 & 24.37 & 149 & 19.87 & Reference & \\
\hline & $\mathrm{G}$ & 537 & 75.63 & 601 & 80.13 & $0.770(0.600,0.986)$ & $0.038^{\mathrm{a}}$ \\
\hline \multirow[t]{2}{*}{ rs1385374 } & $\mathrm{C}$ & 536 & 75.49 & 603 & 80.4 & Reference & \\
\hline & $\mathrm{T}$ & 174 & 24.51 & 147 & 19.6 & $1.332(1.039,1.707)$ & $0.024^{\mathrm{a}}$ \\
\hline \multirow[t]{2}{*}{ rs 10847699} & $\mathrm{C}$ & 172 & 24.36 & 192 & 25.67 & Reference & \\
\hline & $\mathrm{T}$ & 534 & 75.64 & 556 & 74.33 & $1.072(0.845,1.360)$ & 0.566 \\
\hline \multirow[t]{2}{*}{ rs983492 } & $\mathrm{C}$ & 127 & 18.46 & 170 & 22.67 & Reference & \\
\hline & $\mathrm{T}$ & 561 & 81.54 & 580 & 77.33 & $1.295(1.001,1.675)$ & $0.049^{\mathrm{a}}$ \\
\hline \multirow{2}{*}{ rs 1552336} & $\mathrm{C}$ & 637 & 89.72 & 657 & 87.83 & Reference & \\
\hline & $\mathrm{T}$ & 73 & 10.28 & 91 & 12.17 & $0.827(0.597,1.147)$ & 0.256 \\
\hline
\end{tabular}

${ }^{\text {a }}$ Significant association $(p<0.05)$.

CI, confidence interval; OR, odds ratio; SLE, systemic lupus erythematosus; SNP, single-nucleotide polymorphism. 
Table 2. Codominant Analysis of Single-Nucleotide Polymorphisms in SLC15A4 in Systemic Lupus Erythematosus Patients and Controls

\begin{tabular}{|c|c|c|c|c|c|c|c|}
\hline \multirow[b]{2}{*}{$S N P$} & \multirow[b]{2}{*}{ Genotype } & \multicolumn{2}{|c|}{$\operatorname{SLE}(\mathrm{n}=355)$} & \multicolumn{2}{|c|}{ Control $(\mathrm{n}=375)$} & \multirow[b]{2}{*}{ OR $(95 \% C I)$} & \multirow[b]{2}{*}{$\mathrm{P}$} \\
\hline & & Count & $\%$ & Count & $\%$ & & \\
\hline \multirow{3}{*}{ rs7965732 } & AA & 290 & 81.69 & 302 & 80.53 & Reference & \\
\hline & TT & 3 & 0.85 & 6 & 1.6 & $0.521(0.129,2.101)$ & 0.551 \\
\hline & AT & 62 & 17.46 & 67 & 17.87 & $0.964(0.658,1.411)$ & 0.849 \\
\hline \multirow[t]{3}{*}{ rs3765108 } & AA & 154 & 43.38 & 185 & 49.33 & Reference & \\
\hline & GG & 30 & 8.45 & 48 & 12.8 & $0.751(0.454,1.243)$ & 0.264 \\
\hline & AG & 171 & 48.17 & 142 & 37.87 & $1.447(1.063,1.970)$ & $0.019^{\mathrm{a}}$ \\
\hline \multirow{3}{*}{ rs7308691 } & AA & 33 & 9.32 & 49 & 13.14 & Reference & \\
\hline & TT & 167 & 47.18 & 185 & 49.6 & $1.340(0.822,2.185)$ & 0.239 \\
\hline & AT & 154 & 43.5 & 139 & 37.27 & $1.645(1.000,2.705)$ & $0.049^{\mathrm{a}}$ \\
\hline \multirow[t]{3}{*}{ rs7302634 } & $\mathrm{CC}$ & 66 & 18.7 & 76 & 20.27 & Reference & \\
\hline & TT & 112 & 31.73 & 129 & 34.4 & $1.000(0.660,1.515)$ & 0.999 \\
\hline & $\mathrm{CT}$ & 175 & 49.58 & 170 & 45.33 & $1.185(0.801,1.753)$ & 0.394 \\
\hline \multirow{3}{*}{ rs9738216 } & $\mathrm{CC}$ & 66 & 18.64 & 76 & 20.32 & Reference & \\
\hline & TT & 113 & 31.92 & 129 & 34.49 & $1.009(0.666,1.528)$ & 0.967 \\
\hline & $\mathrm{CT}$ & 175 & 49.44 & 169 & 45.19 & $1.192(0.806,1.764)$ & 0.378 \\
\hline \multirow[t]{3}{*}{ rs2291350 } & $\mathrm{CC}$ & 67 & 18.93 & 76 & 20.27 & Reference & \\
\hline & TT & 112 & 31.64 & 129 & 34.4 & $0.985(0.651,1.491)$ & 0.942 \\
\hline & $\mathrm{CT}$ & 175 & 49.44 & 170 & 45.33 & $1.168(0.790,1.725)$ & 0.436 \\
\hline \multirow[t]{3}{*}{ rs11059915 } & GG & 205 & 57.75 & 212 & 56.53 & Reference & \\
\hline & TT & 14 & 3.94 & 25 & 6.67 & $0.579(0.293,1.145)$ & 0.113 \\
\hline & GT & 136 & 38.31 & 138 & 36.8 & $1.019(0.751,1.382)$ & 0.903 \\
\hline \multirow[t]{3}{*}{ rs12298615 } & AA & 25 & 7.06 & 17 & 45.33 & Reference & \\
\hline & GG & 210 & 59.32 & 245 & 65.33 & $0.583(0.306,1.109)$ & 0.097 \\
\hline & AG & 119 & 33.62 & 113 & 30.13 & $0.716(0.367,1.396)$ & 0.326 \\
\hline \multirow[t]{3}{*}{ rs4760592 } & $\mathrm{CC}$ & 112 & 31.55 & 130 & 34.76 & Reference & \\
\hline & GG & 66 & 18.59 & 75 & 20.05 & $1.021(0.674,1.549)$ & 0.92 \\
\hline & CG & 177 & 49.86 & 169 & 45.19 & $1.216(0.875,1.689)$ & 0.245 \\
\hline \multirow{3}{*}{ rs959989 } & AA & 208 & 48.59 & 244 & 65.24 & Reference & \\
\hline & TT & 26 & 7.32 & 17 & 4.55 & $1.794(0.947,3.398)$ & 0.07 \\
\hline & AT & 121 & 34.08 & 113 & 30.22 & $1.256(0.916,1.723)$ & 0.157 \\
\hline \multirow[t]{3}{*}{ rs959987 } & $\mathrm{CC}$ & 237 & 66.95 & 228 & 60.96 & Reference & \\
\hline & TT & 13 & 3.67 & 22 & 5.88 & $0.568(0.280,1.156)$ & 0.115 \\
\hline & $\mathrm{CT}$ & 104 & 29.38 & 124 & 33.16 & $0.807(0.587,1.109)$ & 0.185 \\
\hline rs7311875 & AA & 200 & 56.66 & 211 & 56.27 & Reference & \\
\hline & GG & 16 & 4.53 & 27 & 7.2 & $0.625(0.327,1.195)$ & 0.152 \\
\hline & AG & 137 & 38.81 & 137 & 36.53 & $1.055(0.777,1.432)$ & 0.731 \\
\hline rs11059925 & AA & 221 & 63.87 & 234 & 62.4 & Reference & \\
\hline & $\mathrm{CC}$ & 9 & 2.6 & 16 & 4.27 & $0.596(0.258,1.376)$ & 0.221 \\
\hline & $\mathrm{AC}$ & 116 & 33.53 & 125 & 33.33 & $0.983(0.719,1.343)$ & 0.912 \\
\hline rs10847697 & AA & 26 & 7.32 & 18 & 4.8 & Reference & \\
\hline & GG & 208 & 58.59 & 244 & 65.07 & $0.590(0.315,1.107)$ & 0.097 \\
\hline & AG & 121 & 34.08 & 113 & 30.13 & $0.741(0.386,1.425)$ & 0.368 \\
\hline rs1385374 & $\mathrm{CC}$ & 207 & 58.31 & 246 & 65.6 & Reference & \\
\hline & TT & 26 & 7.32 & 18 & 4.8 & $1.717(0915,3.219)$ & 0.089 \\
\hline & $\mathrm{CT}$ & 122 & 34.37 & 111 & 29.6 & $1.306(0.952,1.793)$ & 0.098 \\
\hline rs10847699 & $\mathrm{CC}$ & 17 & 4.82 & 28 & 7.49 & Reference & \\
\hline & TT & 198 & 56.09 & 210 & 56.15 & $1.553(0.824,2.925)$ & 0.17 \\
\hline & $\mathrm{CT}$ & 138 & 39.09 & 136 & 36.36 & $1.671(0.875,3.193)$ & 0.117 \\
\hline rs983492 & $\mathrm{CC}$ & 13 & 3.78 & 23 & 6.13 & Reference & \\
\hline & TT & 230 & 66.86 & 228 & 60.8 & $1.785(0.882,3.610)$ & 0.103 \\
\hline & $\mathrm{CT}$ & 101 & 29.36 & 124 & 33.07 & $1.441(0.695,2.988)$ & 0.324 \\
\hline rs 1552336 & $\mathrm{CC}$ & 287 & 80.85 & 287 & 76.74 & Reference & \\
\hline & TT & 5 & 1.41 & 4 & 1.07 & $1.250(0.332,4.702)$ & 1 \\
\hline & $\mathrm{CT}$ & 63 & 17.75 & 83 & 22.2 & $0.759(0.526,2.095)$ & 0.139 \\
\hline
\end{tabular}

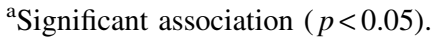


Eighteen SNPs were selected based on a tagger SNP selection algorithm using the default settings in Haploview 4.2 or associations with SLE that have been reported in previous studies (Han et al., 2009). In addition, all SNPs came from the international HapMap project databank for the Han Chinese population of Beijing, China, with a minor allele frequency $>0.05$. For genotyping the 18 SNPs with appropriate methods, 16 of the 18 SNPs were detected by matrix-assisted laser desorption/ionization time-of-flight mass spectrometry (MALDI-TOF MS), with an SNP sequence-specific extension primary added into the polymerase chain reaction (PCR) amplification products (Sangon Biotech). By using a MassARRAY Nanodispenser (Sequenom), the completed genotyping reactions were spotted onto a 384-well SpectroCHIP (Sequenom) and measured using transient $\left(10^{-9} \mathrm{~ns}\right)$ strong laser excitation. Genotype determination was performed in real time with MassARRAY RT software version 3.0.0.4 and analyzed using the MassARRAY Typer software version 3.4 (Sequenom). The genotyping success rate was $>95 \%$.
Genotyping of the remaining two SNPs (rs 7965732, rs 3765108) was performed using TaqMan SNP Genotyping Assays on a LightCycler480 real-time PCR system (Roche). Amplification was performed in a $20-\mu \mathrm{L}$ reaction volume consisting of $10 \mu \mathrm{L} 2 \times$ TaqMan Master Mix, $1 \mu \mathrm{L} 20 \times$ Primer and TaqMan Probe (FAM VIC) dye mix, $7 \mu \mathrm{L} \mathrm{ddH}_{2} \mathrm{O}$, and $2 \mu \mathrm{L}$ template DNA. The following thermal cycle conditions were used: prerun at $95^{\circ} \mathrm{C}$ for $4 \mathrm{~min}$; then denaturation at $95^{\circ} \mathrm{C}$ for $15 \mathrm{~s}$; and an annealing step at $60^{\circ} \mathrm{C}$ for $1 \mathrm{~min}$ in each of 40 cycles.

\section{Statistical analysis}

Data were analyzed with SPSS software for windows (version 17.0; SPSS). The Hardy-Weinberg equilibrium test was applied to examine the genotype frequency of each SNP, using the exact chi-squared test for both the SLE patients and the healthy controls. Linkage disequilibrium blocks of all SNPs were analyzed with Haploview. Conditional logistic regression analysis was applied to evaluate the independent

Table 3. Dominant Analysis of Single-Nucleotide Polymorphisms in SLC15A4 in Systemic Lupus Erythematosus Patients and Controls

\begin{tabular}{|c|c|c|c|c|c|c|c|}
\hline \multirow[b]{2}{*}{$S N P$} & \multirow[b]{2}{*}{ Genotype } & \multicolumn{2}{|c|}{$S L E(\mathrm{n}=355)$} & \multicolumn{2}{|c|}{ Control $(\mathrm{n}=375)$} & \multirow[b]{2}{*}{ OR $(95 \% C I)$} & \multirow[b]{2}{*}{$\mathrm{P}$} \\
\hline & & Count & $\%$ & Count & $\%$ & & \\
\hline \multirow{2}{*}{ rs7965732 } & $\mathrm{TT}+\mathrm{AT}$ & 65 & 18.31 & 73 & 19.47 & $0.927(0.640,1.344)$ & 0.69 \\
\hline & AA & 290 & 81.69 & 302 & 80.53 & Reference & \\
\hline \multirow{2}{*}{ rs3765108 } & $\mathrm{GG}+\mathrm{AG}$ & 201 & 56.62 & 190 & 50.67 & $1.271(0.949,1.701)$ & 0.107 \\
\hline & AA & 154 & 43.38 & 185 & 49.33 & Reference & \\
\hline \multirow{2}{*}{ rs7308691 } & $\mathrm{TT}+\mathrm{AT}$ & 321 & 90.68 & 324 & 86.86 & $1.471(0.922,2.348)$ & 0.104 \\
\hline & AA & 33 & 9.32 & 49 & 13.14 & Reference & \\
\hline \multirow{2}{*}{ rs7302634 } & $\mathrm{TT}+\mathrm{CT}$ & 287 & 81.3 & 299 & 79.73 & $1.105(0.765,1.596)$ & 0.593 \\
\hline & $\mathrm{CC}$ & 66 & 18.7 & 76 & 20.27 & Reference & \\
\hline \multirow{2}{*}{ rs9738216 } & $\mathrm{TT}+\mathrm{CT}$ & 288 & 81.36 & 298 & 79.68 & $1.113(0.771,1.607)$ & 0.568 \\
\hline & $\mathrm{CC}$ & 66 & 18.64 & 76 & 20.32 & Reference & \\
\hline \multirow{2}{*}{ rs2291350 } & $\mathrm{TT}+\mathrm{CT}$ & 287 & 81.07 & 299 & 79.73 & $1.089(0.755,1.570)$ & 0.649 \\
\hline & $\mathrm{CC}$ & 67 & 18.93 & 76 & 20.27 & Reference & \\
\hline \multirow{2}{*}{ rs11059915 } & $\mathrm{TT}+\mathrm{GT}$ & 150 & 42.25 & 163 & 43.47 & $0.952(0.710,1.276)$ & 0.741 \\
\hline & GG & 205 & 57.75 & 212 & 56.53 & Reference & \\
\hline \multirow[t]{2}{*}{ rs12298615 } & $\mathrm{GG}+\mathrm{AG}$ & 329 & 92.94 & 358 & 95.47 & $0.625(0.331,1.178)$ & 0.143 \\
\hline & AA & 25 & 7.06 & 17 & 4.53 & Reference & \\
\hline \multirow[t]{2}{*}{ rs4760592 } & $\mathrm{GG}+\mathrm{CG}$ & 243 & 68.45 & 244 & 65.24 & $1.156(0.849,1.574)$ & 0.358 \\
\hline & $\mathrm{CC}$ & 112 & 31.55 & 130 & 34.76 & Reference & \\
\hline \multirow[t]{2}{*}{ rs959989 } & $\mathrm{TT}+\mathrm{AT}$ & 147 & 41.41 & 130 & 34.76 & $1.326(0.983,1.790)$ & 0.065 \\
\hline & AA & 208 & 58.59 & 244 & 65.24 & Reference & \\
\hline \multirow[t]{2}{*}{ rs959987 } & $\mathrm{TT}+\mathrm{CT}$ & 117 & 33.05 & 146 & 39.04 & $0.771(0.569,1.044)$ & 0.093 \\
\hline & $\mathrm{CC}$ & 237 & 66.95 & 228 & 60.96 & Reference & \\
\hline \multirow[t]{2}{*}{ rs7311875 } & $\mathrm{GG}+\mathrm{AG}$ & 153 & 43.34 & 164 & 43.73 & $0.984(0.734,1.320)$ & 0.915 \\
\hline & AA & 200 & 56.66 & 211 & 56.27 & Reference & \\
\hline \multirow[t]{2}{*}{ rs11059925 } & $\mathrm{CC}+\mathrm{AC}$ & 125 & 36.13 & 141 & 37.6 & $0.939(0.693,1.271)$ & 0.682 \\
\hline & AA & 221 & 63.87 & 234 & 62.4 & Reference & \\
\hline \multirow[t]{2}{*}{ rs10847697 } & $\mathrm{GG}+\mathrm{AG}$ & 329 & 92.68 & 357 & 95.2 & $0.638(0.343,1.185)$ & 0.152 \\
\hline & AA & 26 & 7.32 & 18 & 4.8 & Reference & \\
\hline \multirow[t]{2}{*}{ rs 1385374} & $\mathrm{TT}+\mathrm{CT}$ & 148 & 41.69 & 129 & 34.4 & $1.1363(1.010,1.840)$ & $0.042^{\mathrm{a}}$ \\
\hline & $\mathrm{CC}$ & 207 & 58.31 & 246 & 65.6 & Reference & \\
\hline \multirow[t]{2}{*}{ rs10847699 } & $\mathrm{TT}+\mathrm{CT}$ & 336 & 95.18 & 346 & 92.51 & $1.599(0.860,2.976)$ & 0.135 \\
\hline & $\mathrm{CC}$ & 17 & 4.82 & 28 & 7.49 & Reference & \\
\hline \multirow[t]{2}{*}{ rs983492 } & $\mathrm{TT}+\mathrm{CT}$ & 331 & 96.22 & 352 & 93.87 & $1.664(0.829,3.338)$ & 0.148 \\
\hline & $\mathrm{CC}$ & 13 & 3.78 & 23 & 6.13 & Reference & \\
\hline \multirow[t]{2}{*}{ rs 1552336} & $\mathrm{TT}+\mathrm{CT}$ & 68 & 19.15 & 87 & 23.26 & $0.782(0.547,1.117)$ & 0.176 \\
\hline & $\mathrm{CC}$ & 287 & 80.85 & 287 & 76.74 & Reference & \\
\hline
\end{tabular}

\footnotetext{
${ }^{\mathrm{a}}$ Significant association $(p<0.05)$.
} 
effect of the associated SNPs. The allele, genotype, and haplotype frequency distributions between the SLE patients and healthy individuals were assessed with the chi-squared test and Fisher's exact test. A post hoc power analysis was conducted using Power Analysis and Sample Size (PASS) software (NCSS Statistical Software). Estimated odds ratios (ORs) and 95\% confidence intervals (CIs) were also calculated based on logistic regression. All the tests were two sided and $p$-values $<0.05$ were regarded as significant.

\section{Results}

\section{Allele frequency distribution of SLC15A4 polymorphism}

The ages and genders of the patient and control groups were statistically similar. All polymorphic frequencies were confirmed to be in the Hardy-Weinberg equilibrium in both groups.

The distribution of allele frequencies of 5 of the 18 SNPs from SLC15A4 was significantly different between the groups (Table 1). Specifically, the following SNPs were associated with an increased risk of SLE: rs959989 T (OR 1.317, 95\% CI 1.027-1.689), rs1385374 T (OR 1.332, 95\% CI 1.039-1.707), and rs983492 T (OR 1.295, 95\% CI 1.0011.675). The post hoc calculated statistical power of these SNPs (rs959989 T, rs1385374 T, and rs983492 T) was $58.5 \%, 62.0 \%$, and $51.1 \%$, respectively.

Associated with a decreased risk of SLE were rs12298615 G (OR 0.778, 95\% CI 0.606-0.998) and rs10847697 G (OR $0.770,95 \%$ CI $0.600-0.986)$. We performed a post hoc power analysis and found that the statistical power of rs $12298615 \mathrm{G}$ was $50.1 \%$ and that of rs $10847697 \mathrm{G}$ was $54.3 \%$, with a $5 \%$ type-1 error rate.

\section{Genotype frequency distribution of SLC15A4 polymorphism}

For better understanding the effect of the SNP genotype on the risk of SLE, the genotype distributions of the SLE and control groups were compared using codominant, dominant, and recessive models (Tables $2-4$, respectively). The results

Table 4. Recessive Analysis of Single-Nucleotide Polymorphisms in SLC15A4 in Systemic Lupus Erythematosus Patients and Controls

\begin{tabular}{|c|c|c|c|c|c|c|c|}
\hline \multirow[b]{2}{*}{$S N P$} & \multirow[b]{2}{*}{ Genotype } & \multicolumn{2}{|c|}{$S L E(\mathrm{n}=355)$} & \multicolumn{2}{|c|}{ Control $(\mathrm{n}=375)$} & \multirow[b]{2}{*}{ OR $(95 \% C I)$} & \multirow[b]{2}{*}{$\mathrm{p}$} \\
\hline & & Count & $\%$ & Count & $\%$ & & \\
\hline \multirow{2}{*}{ rs7965732 } & TT & 3 & 0.85 & 6 & 1.6 & $0.524(1.3,2.112)$ & 0.556 \\
\hline & $\mathrm{AA}+\mathrm{AT}$ & 352 & 99.15 & 369 & 98.4 & Reference & \\
\hline \multirow[t]{2}{*}{ rs3765108 } & GG & 30 & 8.45 & 48 & 12.8 & $0.629(0.389,1.018)$ & 0.057 \\
\hline & $\mathrm{AA}+\mathrm{AG}$ & 325 & 91.55 & 327 & 82.7 & Reference & \\
\hline \multirow{2}{*}{ rs7308691 } & TT & 167 & 47.18 & 185 & 49.6 & $0.908(0.678,1.214)$ & 0.514 \\
\hline & $\mathrm{AA}+\mathrm{AT}$ & 187 & 52.82 & 188 & 50.4 & Reference & \\
\hline \multirow[t]{2}{*}{ rs7302634 } & TT & 112 & 31.73 & 129 & 34.4 & $0.886(0.65,1.207)$ & 0.444 \\
\hline & $\mathrm{CC}+\mathrm{CT}$ & 241 & 68.27 & 246 & 65.6 & Reference & \\
\hline \multirow[t]{2}{*}{ rs9738216 } & TT & 113 & 31.92 & 129 & 34.49 & $0.891(0.654,1.213)$ & 0.462 \\
\hline & $\mathrm{CC}+\mathrm{CT}$ & 241 & 68.08 & 245 & 65.51 & Reference & \\
\hline \multirow[t]{2}{*}{ rs2291350 } & TT & 112 & 31.64 & 129 & 34.4 & $0.883(0.648,1.202)$ & 0.428 \\
\hline & $\mathrm{CC}+\mathrm{CT}$ & 242 & 68.36 & 246 & 65.6 & Reference & \\
\hline \multirow[t]{2}{*}{ rs11059915 } & TT & 14 & 3.94 & 25 & 6.67 & $0.575(0.294,1.124)$ & 0.102 \\
\hline & GG+GT & 341 & 96.06 & 350 & 93.33 & Reference & \\
\hline \multirow[t]{2}{*}{ rs12298615 } & GG & 210 & 59.32 & 245 & 65.33 & $0.774(0.573,1.045)$ & 0.094 \\
\hline & $\mathrm{AA}+\mathrm{AG}$ & 144 & 40.68 & 130 & 34.66 & Reference & \\
\hline \multirow[t]{2}{*}{ rs4760592 } & GG & 66 & 18.59 & 75 & 20.05 & $0.910(0.630,1.316)$ & 0.617 \\
\hline & $\mathrm{CC}+\mathrm{CG}$ & 289 & 81.41 & 299 & 79.95 & Reference & \\
\hline \multirow[t]{2}{*}{ rs959989 } & TT & 26 & 7.32 & 17 & 4.55 & $1.660(0.884,3.114)$ & 0.111 \\
\hline & $\mathrm{AA}+\mathrm{AT}$ & 329 & 92.68 & 357 & 95.45 & Reference & \\
\hline \multirow[t]{2}{*}{ rs959987 } & TT & 13 & 3.67 & 22 & 5.88 & $0.610(0.302,1.230)$ & 0.167 \\
\hline & $\mathrm{CC}+\mathrm{CT}$ & 341 & 96.33 & 352 & 94.12 & Reference & \\
\hline \multirow[t]{2}{*}{ rs7311875 } & GG & 16 & 4.53 & 27 & 7.2 & $0.612(0.324,1.156)$ & 0.127 \\
\hline & $\mathrm{AA}+\mathrm{AG}$ & 337 & 95.47 & 348 & 92.8 & Reference & \\
\hline \multirow[t]{2}{*}{ rs11059925 } & $\mathrm{CC}$ & 9 & 2.6 & 16 & 4.27 & $0.599(0.261,1.374)$ & 0.222 \\
\hline & $\mathrm{AA}+\mathrm{AC}$ & 337 & 97.4 & 359 & 95.73 & Reference & \\
\hline \multirow[t]{2}{*}{ rs10847697 } & GG & 208 & 58.59 & 244 & 65.07 & $0.760(0.563,1.025)$ & 0.072 \\
\hline & $\mathrm{AA}+\mathrm{AG}$ & 147 & 41.41 & 131 & 34.93 & Reference & \\
\hline \multirow[t]{2}{*}{ rs1385374 } & TT & 26 & 7.32 & 18 & 4.8 & $1.567(0.844,2.912)$ & 0.152 \\
\hline & $\mathrm{CC}+\mathrm{CT}$ & 329 & 92.68 & 357 & 95.2 & Reference & \\
\hline \multirow[t]{2}{*}{ rs10847699 } & TT & 198 & 56.09 & 210 & 56.15 & $0.998(0.744,1.337)$ & 0.987 \\
\hline & $\mathrm{CC}+\mathrm{CT}$ & 155 & 43.91 & 164 & 43.85 & Reference & \\
\hline \multirow[t]{2}{*}{ rs983492 } & TT & 230 & 66.86 & 228 & 60.8 & $1.301(0.958,1.766)$ & 0.091 \\
\hline & $\mathrm{CC}+\mathrm{CT}$ & 114 & 33.14 & 147 & 39.2 & Reference & \\
\hline \multirow[t]{2}{*}{ rs 1552336} & & 5 & 1.41 & 4 & 1.07 & $1.321(0.352,4.961)$ & 0.937 \\
\hline & $\mathrm{CC}+\mathrm{CT}$ & 350 & 98.59 & 370 & 98.93 & Reference & \\
\hline
\end{tabular}


Table 5. Conditional Logistic Regression Analysis of SLC15A4 Single-Nucleotide Polymorphisms Associated with Systemic Lupus Erythematosus

\begin{tabular}{lcccccc}
\hline & & \multicolumn{5}{c}{ padjusted when conditioned on: } \\
\cline { 3 - 7 } & $\mathrm{p}^{\mathrm{a}}$ & rs12298165 & rs959989 & rs10847697 & rs1385374 & rs983492 \\
\hline rs12298165 & 0.056 & N/A & 0.738 & 0.874 & 0.355 & 0.129 \\
rs959989 & 0.036 & 0.146 & N/A & 0.109 & 0.136 & 0.101 \\
rs10847697 & 0.045 & 0.895 & 0.99 & N/A & 0.99 & 0.124 \\
rs1385374 & 0.029 & 0.222 & 0.313 & 0.99 & N/A & 0.085 \\
rs983492 & 0.055 & 0.16 & 0.155 & 0.155 & 0.172 & N/A \\
\hline
\end{tabular}

${ }^{\mathrm{a}} p$-Value, calculated by logistic regression analysis under the additive model.

N/A, not applicable.

of the codominant model (Table 2) indicate that in both the SLE and control groups, the frequency distribution of the AG genotype of rs3765108 was significantly higher than that of the AA genotype $(p=0.019$, OR 1.447 , 95\% CI $1.063-$ 1.970), and the frequency distribution of the AT genotype of rs7308691 was significantly higher than that of the AA genotype ( $p=0.049$, OR 1.645, 95\% CI 1.000-2.705).

According to the analysis using the dominate model (Table 3), only one SNP (rs1385374, TT+CT cf.CC, $p=0.042$, OR $1.1363,95 \%$ CI 1.010-1.840) was significantly associated with increased risk of SLE. According to the analysis results of the recessive model, there were no significant differences among the 18 SNP genotypes (Table 4).

\section{Linkage disequilibrium analysis}

To confirm that the five detected SNPs (rs959989, rs1385374, rs983492, rs12298615, and rs10847697) are independent, we performed a conditional analysis (Table 5). However, the analysis showed complete abrogation of genetic associations among the five SNPs. This may be due to the relatively high $p$-value before the analysis, due to the small sample size. We found that the association of rs959989 and rs983492 was slightly attenuated, while associations among rs12298615, rs10847697, and rs138537 were markedly attenuated when adjusted for each other (Table 5). In addition, using Haploview, the four SNPs rs12298615, rs959989, rs10847697, and rs 138537 are in near absolute linkage disequilibrium $\left(\mathrm{r}^{2}>\right.$ 0.98). Thus, we chose rs959989 from the four SNPs and rs983492 to perform a linkage disequilibrium analysis. Future studies with a large sample are warranted. We also note that other variants not assessed in this study are possible and might contribute to this effect.

Next, we performed linkage disequilibrium with the two SNPs rs959989 and rs983492 by using Haploview (Fig. 1). Table 6 shows the frequencies of different haplotypes and the results from the analysis of the association with SLE. The prevalence of the haplotype TA was significantly higher in the SLE patients than in the control group $(p=0.024$, OR $1.333,95 \%$ CI 1.039-1.125).

\section{Discussion}

In this study, we investigated 18 SNPs in the SLC15A4 gene with a case-control study and found that five SNPs (rs959989, rs1385374, rs983492, rs12298615, and rs10847697) were associated with SLE in this Han Chinese population. Using a codominant model for analysis, we found that the frequencies of the rs3765108 AG genotype and the rs7308691 AT genotype were significantly higher in the SLE patients, suggesting that these SNPs may be a risk factor for SLE. Moreover, by using the dominant model, the results suggest that rs1385374 (TT+CT cf. CC) was associated with increased risk of SLE. In addition, one SLC15A4 haplotype was also associated with SLE.

SLE is a systemic autoimmune disease accompanied by auto-antibodies, including anti-DNA and anti-small nuclear ribonucleoprotein (snRNP) antibodies, which lead to multiple organ damage (Liu and Davidson, 2012). Nucleic acid binding receptors such as TLR7 and TLR9 are crucial in SLE, promoting the production of IFN-1 through plasmacytoid dendritic cells and activating autoreactive B cells (Cao et al., 2008; Green and Marshak-Rothstein, 2011). Moreover,

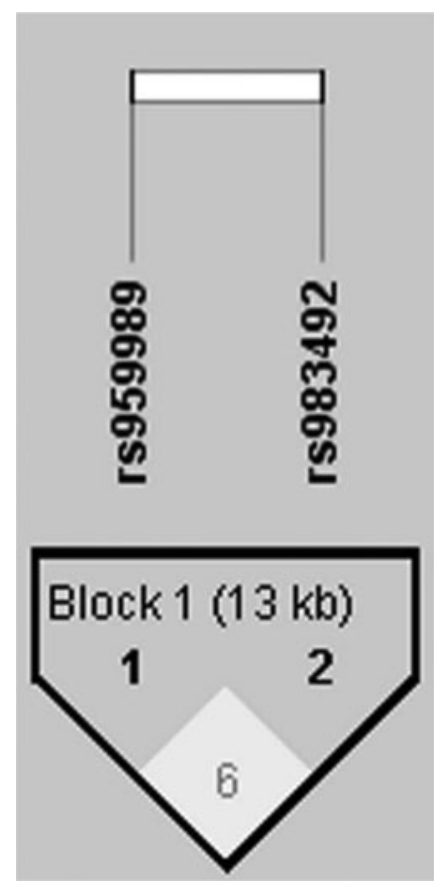

FIG. 1. Analysis of linkage disequilibrium of two singlenucleotide polymorphisms in SLC15A4 in systemic lupus erythematosus patients and controls. The haplotype block structure was analyzed using Haploview software, version 4.2. Each box gives the coefficient of determination $\left(r^{2}\right)$, and the $r^{2}$ color scheme is as follows: white $\left(r^{2}=0\right)$, gray $\left(0<r^{2}\right.$ $<1)$, black $\left(r^{2}=1\right)$. 
Table 6. Haplotype Analysis in Systemic Lupus Erythematosus Patients and Controls

\begin{tabular}{lccccc}
\hline Haplotype & Total $(\%)$ & Case $(\%)$ & Control $(\%)$ & $\mathrm{p}$ & OR (95\% CI) \\
\hline AT & $840(57.5)$ & $406(57.2)$ & $434(57.9)$ & 0.792 & $0.972(0.790-1.197)$ \\
TA & $318(21.8)$ & $173(24.4)$ & $146(19.5)$ & $0.024^{\mathrm{a}}$ & $1.333(1.039-1.125)$ \\
AC & $299(20.5)$ & $131(18.5)$ & $168(22.4)$ & 0.062 & $0.784(0.607-1.012)$ \\
\hline
\end{tabular}

${ }^{\text {a }}$ Significant association $(p<0.05)$.

TLR7 and IFN- $\alpha$ can act together and induce the switching of B cells to IgG2a/c during influenza infection (Marshak-Rothstein, 2006; Heer et al., 2007). On the contrary, SLC15A4 was found to be required for the production of antibodies in $\mathrm{B}$ cells in a mouse model of lupus, and the lack of SLC15A4 in B cells led to reduced production of IgG2a and IgG2c auto-antibodies such as anti-snRNP and anti-DNA antibodies (Kobayashi et al., 2014). Interestingly, Dosenovic et al. (Dosenovic et al., 2015) demonstrated that a functional SLC15A4 was required for effective antibody isotype switching to $\operatorname{IgG} 2 \mathrm{c}$, in response to TLR9 stimulation.

Previous studies (Lee et al., 2009; Blasius et al., 2010; Sasawatari et al., 2011; Baccala et al., 2013) reported that SLC15A4 has a role in maintaining the appropriate endosomal $\mathrm{pH}$ and is necessary for the innate immune response triggered by NOD1, NOD2, TLR7, and TLR9. In a feeble mouse model, defective SLC15A4 led to chronic viral infection by reducing the function of plasmacytoid dendritic cells (Blasius et al., 2012). Previous studies showed that synonymous mutants can affect splicing events, messenger RNA stability, microRNA binding, nucleosome formation, and genes' translational efficiency, sometimes even causing disorders (Chamary et al., 2006; Plotkin and Kudla, 2011; Waldman et al., 2011). In our study, we found a significantly lower risk of SLE associated with the SNP rs10847697, a synonymous polymorphism that has been reported associated with discoid rash or renal disorder (He et al., 2010; Wang et al., 2013). Therefore, this will give us a new research direction to investigate the influence of rs10847697 on the function of SLC15A4. The others four SNPs, however, are all located in the intron region and it is hard to investigate their functional studies.

The first GWAS regarding SLC15A4 showed that it is closely associated with type 2 diabetes in Japan (Takeuchi et al., 2008). Subsequently, another GWAS by Han et al. reported that $S L C 15 A 4$ is a new susceptibility gene for SLE and identified two SNPs (rs10847697, rs1385374) associated with SLE in a Chinese population. This is in agreement with our results. In addition, several other research projects revealed that many clinical features were associated with the two SNPs (He et al., 2010; Wang et al., 2013; Zuo et al., 2014).

Interestingly, a recent study identified new risk loci associated with SLE, using high-density genotyping based on the Immunochip SNP microarray, in Asian ancestry (Sun et al., 2016). In their research, five SNPs (rs959989, rs1385374, rs983492, rs12298615, and rs10847697) were detected and are generally consistent with our results, except rs983492. We speculate that this difference may be due to different detection methods and racial constitution. Further studies with larger sample sizes are needed to confirm this hypothesis.
The present study is limited in that the $p$-values reflected much less significance compared with previous studies, and no significant difference was indicated after the Bonferroni correction or through the Benjamini-Hochberg method. This may be due to the relatively small sample size of our study (hundreds), compared with previous studies (thousands), which limited statistical power. It also must be noted that patients of other ethnicities may have greater differences in allele frequencies (Mori et al., 2005) than our Han Chinese population. Therefore, our results may not be applicable to other populations. Functional studies of the identified SNPs are warranted, and we are planning a future investigation of the expression and $3^{\prime}$-untranslated region of the gene using microRNAs in vitro.

The pathogenesis of SLE is believed to involve the combined effects of a large number of minor genes, and one of these is $S L C 15 A 4$. Our study provides clues that help elucidate the role of the SLC15A4 gene in the pathogenesis of SLE in Han Chinese people. More comprehensive research that includes populations of different ethnicities is warranted. Further studies should focus on the physiological functions of the mutated loci that occur with significantly different frequencies between SLE and healthy individuals, as well as the influence of SNPs in SLC15A4 on SLE in the Han Chinese population.

\section{Acknowledgments}

This work was supported by the National Natural Science Foundation of China (No. 81301369) and the Clinical Innovation Foundation of the Third Military Medical University (SWH2001LC012).

\section{Author Disclosure Statement}

No competing financial interests exist.

\section{References}

Baccala R, Gonzalez-Quintial R, Blasius AL, et al. (2013) Essential requirement for IRF8 and SLC15A4 implicates plasmacytoid dendritic cells in the pathogenesis of lupus. Proc Natl Acad Sci U S A 110:2940-2945.

Blasius AL, Arnold CN, Georgel P, et al. (2010) Slc15a4, AP-3, and Hermansky-Pudlak syndrome proteins are required for Toll-like receptor signaling in plasmacytoid dendritic cells. Proc Natl Acad Sci U S A 107:19973-19978.

Blasius AL, Krebs P, Sullivan BM, et al. (2012) Slc15a4, a gene required for $\mathrm{pDC}$ sensing of TLR ligands, is required to control persistent viral infection. PLoS Pathog 8:e1002915.

Cao W, Manicassamy S, Tang H, et al. (2008) Toll-like receptor-mediated induction of type I interferon in plasmacytoid dendritic cells requires the rapamycin-sensitive PI(3)K-mTOR-p70S6K pathway. Nat Immunol 9:1157-1164. 
Chamary JV, Parmley JL, Hurst LD (2006) Hearing silence: non-neutral evolution at synonymous sites in mammals. Nat Rev Genet 7:98-108.

Dosenovic P, Adori M, Adams WC, et al. (2015) Slc15a4 function is required for intact class switch recombination to IgG2c in response to TLR9 stimulation. Immunol Cell Biol 93:136-146.

Green NM, Marshak-Rothstein A (2011) Toll-like receptor driven $\mathrm{B}$ cell activation in the induction of systemic autoimmunity. Semin Immunol 23:106-112.

Han JW, Zheng HF, Cui Y, et al. (2009) Genome-wide association study in a Chinese Han population identifies nine new susceptibility loci for systemic lupus erythematosus. Nat Genet 41:1234-1237.

He CF, Liu YS, Cheng YL, et al. (2010) TNIP1, SLC15A4, ETS1, RasGRP3 and IKZF1 are associated with clinical features of systemic lupus erythematosus in a Chinese Han population. Lupus 19:1181-1186.

Heer AK, Shamshiev A, Donda A, et al. (2007) TLR signaling fine-tunes anti-influenza $\mathrm{B}$ cell responses without regulating effector T cell responses. J Immunol 178:2182-2191.

Hochberg MC (1997) Updating the American College of Rheumatology revised criteria for the classification of systemic lupus erythematosus. Arthritis Rheum 40:1725.

Kobayashi T, Shimabukuro-Demoto S, Yoshida-Sugitani R, et al. (2014) The histidine transporter SLC15A4 coordinates mTOR-dependent inflammatory responses and pathogenic antibody production. Immunity 41:375-388.

Lee HS, Kim T, Bang SY, et al. (2014) Ethnic specificity of lupus-associated loci identified in a genome-wide association study in Korean women. Ann Rheum Dis 73:1240-1245.

Lee J, Tattoli I, Wojtal KA, et al. (2009) pH-dependent internalization of muramyl peptides from early endosomes enables Nod1 and Nod2 signaling. J Biol Chem 284:23818-23829.

Liu Z, Davidson A (2012) Taming lupus-a new understanding of pathogenesis is leading to clinical advances. Nat Med 18: 871-882.

Marshak-Rothstein A (2006) Toll-like receptors in systemic autoimmune disease. Nat Rev Immunol 6:823-835.

Mori M, Yamada R, Kobayashi K, et al. (2005) Ethnic differences in allele frequency of autoimmune-disease-associated SNPs. J Hum Genet 50:264-266.

Nakamura N, Lill JR, Phung Q, et al. (2014) Endosomes are specialized platforms for bacterial sensing and NOD2 signalling. Nature 509:240-244.

Plotkin JB, Kudla G (2011) Synonymous but not the same: the causes and consequences of codon bias. Nat Rev Genet $12: 32-42$.
Sasawatari S, Okamura T, Kasumi E, et al. (2011) The solute carrier family $15 \mathrm{~A} 4$ regulates TLR9 and NOD1 functions in the innate immune system and promotes colitis in mice. Gastroenterology 140:1513-1525.

Sun C, Molineros JE, Looger LL, et al. (2016) High-density genotyping of immune-related loci identifies new SLE risk variants in individuals with Asian ancestry Nat Genet48:323-330.

Takeuchi F, Ochiai Y, Serizawa M, et al. (2008) Search for type 2 diabetes susceptibility genes on chromosomes 1q, 3q and 12q. J Hum Genet 53:314-324.

Tsokos GC (2011) Systemic lupus erythematosus. N Engl J Med 365:2110-2121.

Waldman YY, Tuller T, Keinan A, et al. (2011) Selection for translation efficiency on synonymous polymorphisms in recent human evolution. Genome Biol Evol 3:749-761.

Wang C, Ahlford A, Jarvinen TM, et al. (2013) Genes identified in Asian SLE GWASs are also associated with SLE in Caucasian populations. Eur J Hum Genet 21:994-999.

Yamashita T, Shimada S, Guo W, et al. (1997) Cloning and functional expression of a brain peptide/histidine transporter. J Biol Chem 272:10205-10211.

Zuo XB, Sheng YJ, Hu SJ, et al. (2014) Variants in TNFSF4, TNFAIP3, TNIP1, BLK, SLC15A4 and UBE2L3 interact to confer risk of systemic lupus erythematosus in Chinese population. Rheumatol Int 34:459-464.

Address correspondence to: Fei Hao, PhD

Department of Dermatology Southwest Hospital Third Military Medical University No. 29 Gaotanyan Centre Street Shapingba District Chongqing 400038 China

E-mail: haofei62@medmail.com.cn

Zhifang Zhai, PhD Department of Dermatology Southwest Hospital Third Military Medical University No. 29 Gaotanyan Centre Street Shapingba District Chongqing 400038

China

E-mail: zhaizf1004@163.com 